\title{
ABSENCE OF CANTOR SPECTRUM FOR A CLASS OF SCHRÖDINGER OPERATORS
}

\author{
NORBERT RIEDEL
}

\begin{abstract}
It is shown that the complete localization of eigenvectors for the almost Mathieu operator entails the absence of Cantor spectrum for this operator.
\end{abstract}

Among almost periodic Schrödinger operators the almost Mathieu operator has enjoyed the majority of attention in the last ten to fifteen years for the following three reasons: First, it is the simplest nontrivial operator of its kind, and therefore it is deemed to be more accessible than others. Second, subjecting the almost Mathieu operator to the Fourier transformation leads to an operator of the same kind (duality property). Third, and probably most important, depending on the parameters involved, the almost Mathieu operator displays nearly all the conceivable features operators of this kind could have, such as point spectrum, absence of point spectrum, absolute continuous spectrum, singular spectrum, etc. According to most researchers who have contributed to this field and who have stated their opinion, however, the only exception from this pattern seems to concern the nature of the spectrum of the almost Mathieu operator, considered as a bounded selfadjoint operator. As manifested by repeatedly stated conjectures (see [9] for one of the earliest sources and [5] for one of the latest), the almost Mathieu operator is expected to have a nowhere dense spectrum (Cantor spectrum) whenever the operator is not periodic. We shall argue that the absence of Cantor spectrum for the almost Mathieu operator not only does occur, but we shall also identify the reason as to why it happens.

\section{2}

The operator in question is defined on the Hilbert space $\ell^{2}(\mathbb{Z})$ as

$$
(H(\alpha, \beta, \theta) \xi)_{n}=\xi_{n+1}+\xi_{n-1}+2 \beta \cos (2 \pi \alpha n+\theta) \xi_{n},
$$

where $\alpha, \beta, \theta$ are real constants. We will be concerned exclusively with the case that $\alpha$ is an irrational number. In this case the spectrum $\operatorname{Sp}(\alpha, \beta)$ of $H(\alpha, \beta, \theta)$ does not depend on the parameter $\theta$. For a full set of $\alpha$ 's (in the sense of Lebesgue measure) which are sufficiently badly approximable by rational numbers and for sufficiently large $\beta$, Fröhlich et al. in [3] and Sinai in [10] have shown independently, with quite involved techniques, that $H(\alpha, \beta, \theta)$ has, for almost all $\theta$, a complete set of eigenvectors that decay exponentially. What is actually shown in these papers is that any generalized eigenvector of

Received by the editors July 15, 1992 and, in revised form, December 9, 1992.

1991 Mathematics Subject Classification. Primary 47B39, 47C15; Secondary 31A15. 
$H(\alpha, \beta, \theta)$ which grows sufficiently slowly as $|n|$ approaches infinity decays exponentially. This fact, combined with some other elementary observations, leads us to assume the validity of the following condition for the parameters $\alpha$ and $\beta$ in question:

(L) For every $\chi \in \operatorname{Sp}(\alpha, \beta)$ there is a $\theta$ such that $H(\alpha, \beta, \theta)$ has an eigenvector that decays exponentially as $|n| \rightarrow \infty$.

This condition entails that the average Lyapunov index for the operator $H(\alpha, \beta, \theta)$ takes the constant value $\log |\beta|$ on $\operatorname{Sp}(\alpha, \beta)$. By virtue of the "showless formula", which establishes a connection between the average Lyapunov index and the logarithmic potential associated with the integrated density of states, it follows that $\operatorname{Sp}(\alpha, \beta)$ is a regular compactum (i.e., the Dirichlet problem is solvable in $(\mathbb{C} \cup\{\infty\}) \backslash \operatorname{Sp}(\alpha, \beta)$ for any continuous function on $\operatorname{Sp}(\alpha, \beta)$ ), and the integrated density of states $\tau$ for $H(\alpha, \beta, \theta)$ is the equilibrium distribution of $\operatorname{Sp}(\alpha, \beta)$ (cf. [11] for the potential theoretic terminology being used). The level curves of the conductor potential associated with $\operatorname{Sp}(\alpha, \beta)$ can be identified with the spectra of perturbed almost Mathieu operators.

Theorem 1 [7]. Assume that (L) holds. A complex number $z$ is contained in the spectrum of the operator

$$
\left(H_{\delta}(\alpha, \beta) \xi\right)_{n}=\xi_{n+1}+\xi_{n-1}+\beta\left(\delta e^{2 \pi \alpha n i}+\delta^{-1} e^{-2 \pi \alpha n i}\right) \xi_{n}, \quad \xi \in \ell^{2}(\mathbb{Z}),
$$

if and only if $\int \log |z-s| d \tau(s)=\log |\beta|+|\log | \delta||$.

The gist in the setup of the proof of this theorem is to consider the operators $H_{\delta}(\alpha, \beta)$ as elements of the irrational rotation $C^{*}$-algebra $\mathscr{A}_{\alpha}$ associated with the number $\alpha$. The $C^{*}$-algebra $\mathscr{A}_{\alpha}$ is generated by two unitary operators $u$ and $v$ satisfying the commutation relation $u v=e^{2 \pi \alpha i} v u$. The operator $H_{\delta}(\alpha, \beta)$ can be identified with the element $u+u^{-1}+\beta\left(\delta v+\delta^{-1} v^{-1}\right)$. The resolvent of the operator $H_{\delta}(\alpha, \beta)$ on each connected component of the resolvent set can now be expanded into absolutely convergent series in the monomials $u^{p} v^{q}$. This in turn leads to the definition of subharmonic functions measuring the order of decay for these expansions in every point of the resolvent set. The proof of Theorem 1 is then established by exhibiting interrelationships between these subharmonic functions. It should be noted that the conclusion of Theorem 1 remains true if the condition (L) is replaced by the assumption that $\alpha$ is sufficiently well approximable by rationals and that $|\beta| \geq 1$ [8]. This seems to suggest that the statement in Theorem 1 may be true for all irrational numbers $\alpha$ and for all $|\beta| \geq 1$. By a basic duality argument it is readily seen that a similar characterization of the level curves of the conductor potential associated with $\mathrm{Sp}(\alpha, \beta)$ in terms of the spectra of perturbed almost Mathieu operators holds for any $\beta$ with $0<|\beta|<1$, whenever the statement in Theorem 1 holds for $\beta^{-1}$. The condition (L), in conjunction with Theorem 1 , is indispensible for the following assertion.

Theorem 2 [7]. Assume that (L) holds. Then $\operatorname{Sp}(\alpha, \beta)$ is not a Cantor set.

The issue of the nature of $\operatorname{Sp}(\alpha, \beta)$ has been taken up in a number of papers. Choi et al. [2], inspired by earlier work by Bellissard and Simon [1], 
show that for numbers $\alpha$ which are sufficiently well approximable by rationals, $\operatorname{Sp}(\alpha, \beta)$ is indeed a Cantor set and, moreover, they show that all possible gaps in $\operatorname{Sp}(\alpha, \beta)$ do actually occur. The most penetrating analysis to date of the nature of $\operatorname{Sp}(\alpha, \beta)$ has been conducted by Helffer and Sjöstrand in three memoirs [4]. Most of their investigation is limited to the case $\beta=1$ for $\alpha$ 's which are sufficiently badly approximable by rational numbers. Even though this work produces a wealth of detailed information, it leaves the main question unanswered. In [10] Sinai claims that $\operatorname{Sp}(\alpha, \beta)$ is a Cantor set in those cases where he establishes complete localization of eigenvectors. As we have seen, this claim does not conform with our Theorem 2 .

The proof of Theorem 2 heavily relies on some $C^{*}$-algebraic machinery that was developed in [6] in order to address spectral problems for the almost Mathieu operator. In conjunction with Theorem 1, Theorem 2 provides for an argument which translates the condition (L) into a smoothness condition for the logarithmic potential involved. In this context condition (L) appears as a device to control the growth of the (noncommutative) Fourier expansions of certain functionals associated with points in $\operatorname{Sp}(\alpha, \beta)$. That forementioned smoothness of the logarithmic potential is shown to conflict with the assumption that $\operatorname{Sp}(\alpha, \beta)$ is a Cantor set.

\section{REFERENCES}

1. J. Bellissard and B. Simon, Cantor spectrum for the almost Mathieu equation, J. Funct. Anal. 48 (1982), 408-519.

2. M.-D. Choi, G. A. Elliott, and N. Yui, Gauss polynomials and the rotation algebra, Invent. Math. 99 (1990), 225-246.

3. J. Fröhlich, T. Spencer, and P. Wittwer, Localization for a class of one-dimensional quasiperiodic Schrödinger operators, Comm. Math. Phys. 132 (1990), 5-25.

4. B. Helffer and J. Sjöstrand, Analyse semi-classique pour l'équation de Harper. I-III, Mém. Soc. Math. France (N.S.) (4) 116, (4) 117, (1) 118, (1988-1990).

5. L. Pastur and A. Figotin, Spectra of random and almost-periodic operators, Springer-Verlag, New York, 1992.

6. N. Riedel, Almost Mathieu operators and rotation $C^{*}$-algebras, Proc. London Math. Soc. (3) 56 (1988), 281-302.

7. _ The spectrum of a class of almost periodic operators, preprint.

8. _ Regularity of the spectrum for the almost Mathieu operator, preprint.

9. B. Simon, Almost periodic Schrödinger operators: a review, Adv. in Appl. Math. 3 (1982), 463-490.

10. Ya. G. Sinai, Anderson localization for one-dimensional difference Schrödinger operator with quasiperiodic potential, J. Stat. Phys. 46 (1987), 861-909.

11. M. Tsuji, Potential theory in modern function theory, Chelsea, New York, 1959.

Department of Mathematics, Tulane University, New Orleans, Louisiana 70118 